\title{
Quantum
}

\section{A Uniquely}

Smart Radiation Therapy System

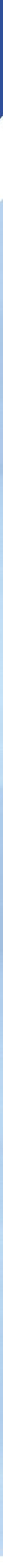

CyberKnife accurar.

Fully integrated Treatment Planning and Delivery

\section{ACCURAY}




\title{
The Crucial Role of the Establishment of Computed Tomography Density Conversion Tables for Treating Brain or Head/Neck Tumors
}

\author{
Shu-Chin Yang ${ }^{1,2(\mathbb{D})}$, Su-Hua Lo ${ }^{3 \text { (D) }}$, Li-Tsuen Shie ${ }^{1(\mathbb{D})}$, Sung-Wei Lee ${ }^{1 \mathbb{1}}$, Sheng-Yow Ho ${ }^{1,4,5}$ \\ ${ }^{1}$ Department of Radiation Oncology, Chi Mei Medical Center, Liouying, Tainan, ${ }^{2}$ Department of Medical Imaging and Radiological Sciences, \\ Kaohsiung Medical University, Kaohsiung, ${ }^{3}$ Chiu Ho Medical System Co., LTD, Taipei, Taiwan, ${ }^{4}$ Graduate Institute of Medical Science, \\ Chang Jung Christian University, ${ }^{5}$ Department of Radiation Oncology, Chi Mei Medical Center, Tainan, Taiwan
}

Received 17 May 2021

Revised 28 July 2021

Accepted 6 August 2021

Corresponding author

Shu-Chin Yang

(fr892072@gmail.com)

Tel: 886-6-6226999

(ext. 71025)

Fax: 886-6-6220226
Purpose: The relationship between computed tomography (CT) number and electron density (ED) has been investigated in previous studies. However, the role of these measures for guiding cancer treatment remains unclear.

Methods: The CT number was plotted against ED for different imaging protocols. The CT number was imported into ED tables for the Pinnacle treatment planning system (TPS) and was used to determine the effect on dose calculations. Conversion tables for radiation dose calculations were generated and subsequently monitored using a dosimeter to determine the effect of different CT scanning protocols and treatment sites. These tables were used to retrospectively recalculate the radiation therapy plans for 41 patients after an incorrect scanning protocol was inadvertently used. The gamma index was further used to assess the dose distribution, percentage dose difference (DD), and distance-to-agreement (DTA).

Results: For densities $<1.1 \mathrm{~g} / \mathrm{cm}^{3}$, the standard deviation of the CT number was $\pm 0.6 \%$ and the greatest variation was noted for brain protocol conditions. For densities $>1.1 \mathrm{~g} / \mathrm{cm}^{3}$, the standard deviation of the CT number was $\pm 21.2 \%$ and the greatest variation occurred for the tube voltage and head and neck $(H \& N)$ protocol conditions. These findings suggest that the factors most affecting the CT number are the tube voltage and treatment site (brain and H\&N). Gamma index analyses for the 41 retrospective clinical cases, as well as brain metastases and H\&N tumors, showed gamma passing rates $>90 \%$ and $<90 \%$ for the passing criterion of $2 \% / 2$ and $1 \% / 1 \mathrm{~mm}$, respectively.

Conclusions: The CT protocol should be carefully decided for TPS. The correct protocol should be used for the corresponding TPS based on the treatment site because this especially affects the dose distribution for brain metastases and $H \& N$ tumor recognition. Such steps could help reduce systematic errors.

Keywords: Computed tomography number, Electron density tables, Dose distribution, Gamma index, Imaging protocols

\section{Introduction}

Computed tomography (CT) is used to determine the ex- tent of a tumor and, subsequently, the treatment planning system (TPS). The CT number depends on various factors such as the tube voltage, field of view (FOV), scanner type,

Copyright $\odot 2021$ Korean Society of Medical Physics

(This is an Open-Access article distributed under the terms of the Creative Commons Attribution Non-Commercial License (http://creativecommons.org/licenses/by$\mathrm{nc} / 4.0$ ) which permits unrestricted non-commercial use, distribution, and reproduction in any medium, provided the original work is properly cited. 
tube current, slice thickness, processing filters, and reconstruction algorithm including artifact reduction [1]. For TPS, tissue density measured using CT is converted to an electron density (ED) to calculate the dose [2]. Thus, the CT number must be accurate because it affects the dose calculation $[3,4]$.

In most radiotherapy departments, the quality of CT images is ensured by daily, monthly, and yearly quality assurance mechanisms. The conversion of the CT number to ED is important because different CT scanning protocols use a unique conversion table to establish ED. The relationship between the CT number and ED has been evaluated in several studies [5]. Many radiotherapy departments use more than one scanning protocol to achieve better image quality assurance. The protocol used depends on the treatment site and the patient's body characteristics. However, regardless of the protocol, most departments use only one conversion table. Thus, the dose calculations may be inaccurate [6].

The relationship between the CT number and ED has been the subject of several studies [5,7-11]. Previous studies have mainly used phantoms to illustrate the relationship between the CT number and ED. There is a concern that varying scanning parameters will change the $\mathrm{CT}$ number of the images and subsequently result in inaccurate dosimetric information in the TPS. In this study, the CT number is plotted against ED for different imaging protocols. All CT scanning protocols in clinical use in the departments of the present study were reviewed to create a specific conversion table for each scanning protocol. In addition, the conversion tables in 41 patients were retrospectively used and the effects on dose calculations were determined.

\section{Materials and Methods}

CT scanning was performed using a GE Discovery ${ }^{\mathrm{TM}}$ CT 590RT scanner (GE Medical Systems, Milwaukee, WI, USA) with a gantry aperture and a standard FOV of 80 and $65 \mathrm{~cm}$, respectively. More than one scanning protocol was used to achieve better image quality, depending on the treatment site and patient's body characteristics.

A cylindrical Virtual Water ${ }^{\mathrm{TM}}$ phantom (Cheese phantom) was used for the different scanning protocols to plot the CT number-ED calibration curve. The phantom contains in-

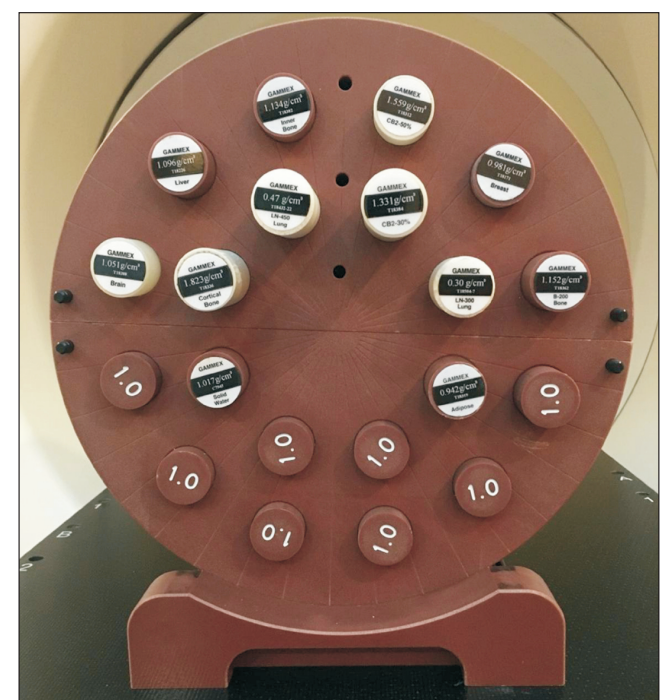

Fig. 1. The cylindrical Cheese Virtual Water ${ }^{\mathrm{TM}}$ phantom.

terchangeable plugs made of various tissue-equivalent materials. The device uses 12 plugs of different densities, each with a diameter and height of 2.7 and $7 \mathrm{~cm}$, respectively (Fig. 1). The physical density (in gram per cubic centimeter) ranges from 0.3 (LN-300 lung) to 1.823 (T18336 cortical bone) for an ED relative to water of 1.107. The phantom was placed in the center of the CT gantry and carefully aligned using lasers. Scanning was performed using different imaging protocols and tube voltages $(80-140 \mathrm{kVp})$, tube currents (300-400 mA), slice thicknesses (5 mm), and FOV (30, 50, and $65 \mathrm{~cm}$ ). A $512 \times 512$ matrix was used to reconstruct the images. The CT scanning protocols for four treatment sites are presented in Table 1.

After image reconstruction, a circular region of interest (ROI) with a diameter of $1.5 \mathrm{~cm}$ was placed on each density plug and the mean CT number for each ROI was recorded. To minimize the effect of image artifacts and beam hardening, multiple CT scans of the phantom were acquired using different combinations of insertion positions and the resultant mean CT numbers were averages. The resultant CT number-ED conversion tables were compared for different tube voltages, tube currents, slice thicknesses, and FOVs as aforementioned.

The CT number-ED conversion tables were imported into Pinnacle TPS (Philips Radiation Oncology Systems, Fitchburg, WI, USA), which transforms the CT number as follows [12]: 
Table 1. Analyzed results of the computed tomography scanning parameters

\begin{tabular}{cccccc}
\hline \multirow{2}{*}{ Scan protocol } & \multicolumn{5}{c}{ Parameter } \\
\cline { 2 - 6 } & Scan technique & Tube voltage $(\mathrm{kVp})$ & Tube current $(\mathrm{mA})$ & Slice thickness $(\mathrm{mm})$ & FOV $(\mathrm{cm})$ \\
\hline Brain & Axial & 80,120 , and 140 & 300 & 5 & 30,50, and 65 \\
Head and neck & Helical & 80,120 , and 140 & 350 & 5 & 30,50, and 65 \\
Chest & Helical & 80,120 , and 140 & 400 & 5 & 30,50, and 65 \\
Pelvic & Helical & 80,120 , and 140 & 400 & 5 & 30,50, and 65 \\
\hline
\end{tabular}

FOV, field of view.

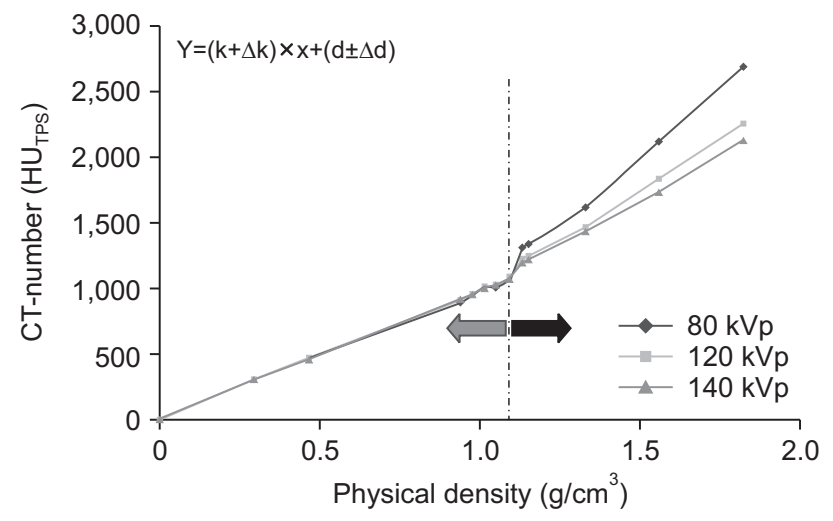

Fig. 2. The computed tomography (CT) number-electron density conversion tables were split into two ranges: $<1.1$ and $>1.1 \mathrm{~g} / \mathrm{cm}^{3}$ densities. A straight-line fit was constructed for each range. The mean and standard deviation for each range and set of scanning conditions were used as a measure of the CT number relative to the CT scanning parameters.

$$
\begin{aligned}
& \mathrm{CT}_{\mathrm{HU}} \leq-1.024, \mathrm{TPS}_{\mathrm{HU}}=0 \\
& -1.024 \leq \mathrm{CT}_{\mathrm{HU}} \leq 4.000 ; \mathrm{TPS}_{\mathrm{HU}}=\mathrm{CT}_{\mathrm{HU}}+1.000
\end{aligned}
$$

The CT number-ED conversion tables for various tube voltages $(80,120$, and $140 \mathrm{kVp})$ are shown in Fig. 2.

A total of 48 CT number-ED conversion tables were produced and the relevant parameters were compared. The obtained curves were divided into two ranges based on Zurl et al. [11]: $<1.1$ and $>1.1 \mathrm{~g} / \mathrm{cm}^{3}$ of densities. A straight-line fit was constructed for each range. The mean and standard deviation for each range and set of scanning conditions were used as a measure of the CT number relative to the CT scanning parameters.

Then, the resulting new conversion tables were used to recalculate the dose distributions from the data of 41 patients, of which 9 exhibited brain metastases, 9 had head and neck cancer $(\mathrm{H} \& \mathrm{~N}), 11$ had lung cancer, and 12 had prostate cancer. Moreover, 123 plans were studied, includ- ing $27,27,33$, and 36 for brain metastases, H\&N tumors, lung tumors, and prostate tumors, respectively. The effect of the CT number on the dose was determined in terms of tube voltage and tumor position. These patients were retrospectively identified for inclusion in the analyses. The Institutional Review Board of the Chi Mei Medical Center, Liouying approved of the study and the approval number was 10811-L03. Informed consent was not required because this study had the lowest risk, and any possible risk to the patient did not exceed compared with those who did not participate in the study.

Of the patients, 12 and 29 received intensity-modulated radiation therapy (IMRT) and volumetric modulated arc therapy (VMAT), respectively. Each plan used a different CT number-ED conversion table for the specific tube voltage $(80,120$, and $140 \mathrm{kVp})$, and the dose distribution was corrected for inhomogeneity. The remaining parameters, such as beam arrangements and monitor unit (MU), were the same.

The gamma index was used to determine the effect on dose calculations for the TPS and was measured using dose distribution, percentage dose difference (DD), and distance-to-agreement (DTA). The distribution was displayed to provide a quantitative assessment of the quality of the calculation for the regions that passed and failed the acceptance criteria [13]. The gamma index produces a passing criterion assigned to each point, for which gamma $\leq 1$ indicates pass and other values indicate failure. The percentage of passing points in the gamma distribution is referred to as passing criteria (or \%GP). Each measured point was evaluated to determine if the percentage DD or DTA exceeded the established passing criterion (i.e., $2 \%$ and $2 \mathrm{~mm}$, respectively) [14-16].

The difference in dose calculations for the gamma index 
(dose distributions, percentage DD, and DTA) for various ED tables was calculated. To compare the four treatment sites (brain metastases, H\&N cancer, lung cancer, and prostate cancer), the MU calculated for the $140 \mathrm{kVp}$ CT scan for optimum coverage of the planning target volume was used to calculate other CT number-ED conversion tables that used different tube voltage settings. The planning parameters, such as the beam arrangements, fields, and MU, were the same.

Each initial plan with a calibration table was used to generate a verification plan in PTW-VeriSoft ${ }^{\circledR}$ to generate the reference two-dimensional dose distribution. Local and global gamma index analyses were used. The local gamma index analysis normalizes the percent difference for every point to the expected dose at each point, whereas the global gamma index analysis normalizes the percent difference for every point to a globally used single value, which is usually the maximum planned dose [17]. Thus, the gamma passing rate calculated using the global gamma index is always higher than or equal to the local gamma index, where the same criteria and a lower dose threshold are used. Both the global and local gamma passing rates with various gamma criteria were $2 \% / 2 \mathrm{~mm}, 1 \% / 1 \mathrm{~mm}$, and $0.5 \% / 0.5 \mathrm{~mm}$. These rates were calculated for the absolute doses using PTWVeriSoft $^{\circledR}$, which linearly interpolates the calculated dose to a resolution $<0.5 \mathrm{~mm}$.

\section{Results}

\section{Computed tomography number-electron density conversion}

Calculations using the Cheese phantom showed different effects for image protocols for 12 different density ranges. For densities $<1.1 \mathrm{~g} / \mathrm{cm}^{3}$, the standard deviation for the CT number was $\pm 0.6 \%$ and the greatest variation was noted for brain protocol conditions. For densities $>1.1 \mathrm{~g} / \mathrm{cm}^{3}$, the standard deviation for the CT number was $\pm 21.2 \%$ and the value varied most significantly with tube voltage and $H \& N$ protocol conditions (Fig. 3).

This is probably due to the artifact in the plug that has different chemical compositions but the same physical density. The differences in CT numbers versus tube volt-

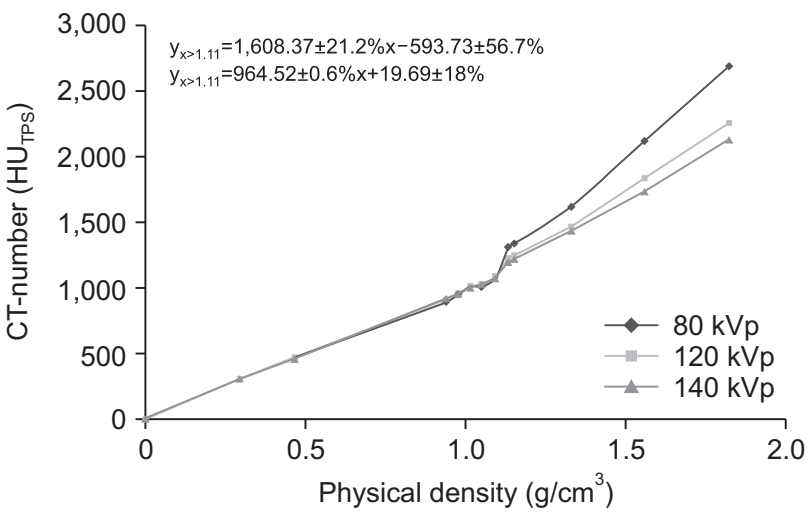

Fig. 3. The computed tomography (CT) number-electron density conversion tables using different tube voltages. For densities $>1.1$ $\mathrm{g} / \mathrm{cm}^{3}$, the standard deviation for the CT number was $\pm 21.2 \%$. For densities $<1.1 \mathrm{~g} / \mathrm{cm}^{3}$, the standard deviation for the CT number was $\pm 0.6 \%$.

ages are minimal in the density region from 0.3 (lung) to 1.0 (water), and no differences were noted in low-density materials (lung) of $4 \%$ (332 $\mathrm{HU}$ at $80 \mathrm{kVp}$ vs. $320 \mathrm{HU}$ at 140 $\mathrm{kVp}$ ). Significant differences in high-density materials (cortical bone) were shown in $28 \%(2,856 \mathrm{HU}$ at $80 \mathrm{kVp}$ vs. 2,223 $\mathrm{HU}$ at $140 \mathrm{kVp}$ ) and a higher tube voltage that resulted in a lower CT number.

The CT number to relative ED calibration curves for the differences in FOV (30, 50, and 65 FOV) was compared. The differences in CT numbers versus FOV are maximum in the density region from 0.3 (lung) to 1.0 (water); the significant differences in CT number were $17 \%$ (371 HU at $30 \mathrm{FOV}$ vs. $317 \mathrm{HU}$ at $65 \mathrm{FOV})$ and $9 \%(2,332 \mathrm{HU}$ at $30 \mathrm{FOV}$ vs. 2,118 at 65 FOV) occurring for low-density materials (lung) and high-density materials (cortical bone), respectively.

Moreover, the CT number-ED tables showed that there is no significant relationship between tube current and slice thickness.

\section{Global gamma passing rates for clinical cases}

Fig. 4 shows the global gamma passing rates for various passing criteria for ED calibrations using 80 and $140 \mathrm{kVp}$ and 120 and $140 \mathrm{kVp}$ for various treatment sites as measured using a dose distribution dosimeter with PTW-VeriSoft ${ }^{\circledR}$ measurements.

The comparison of ED calibrations for 80 and $140 \mathrm{kVp}$ for the four tumor sites showed the lowest gamma passing 


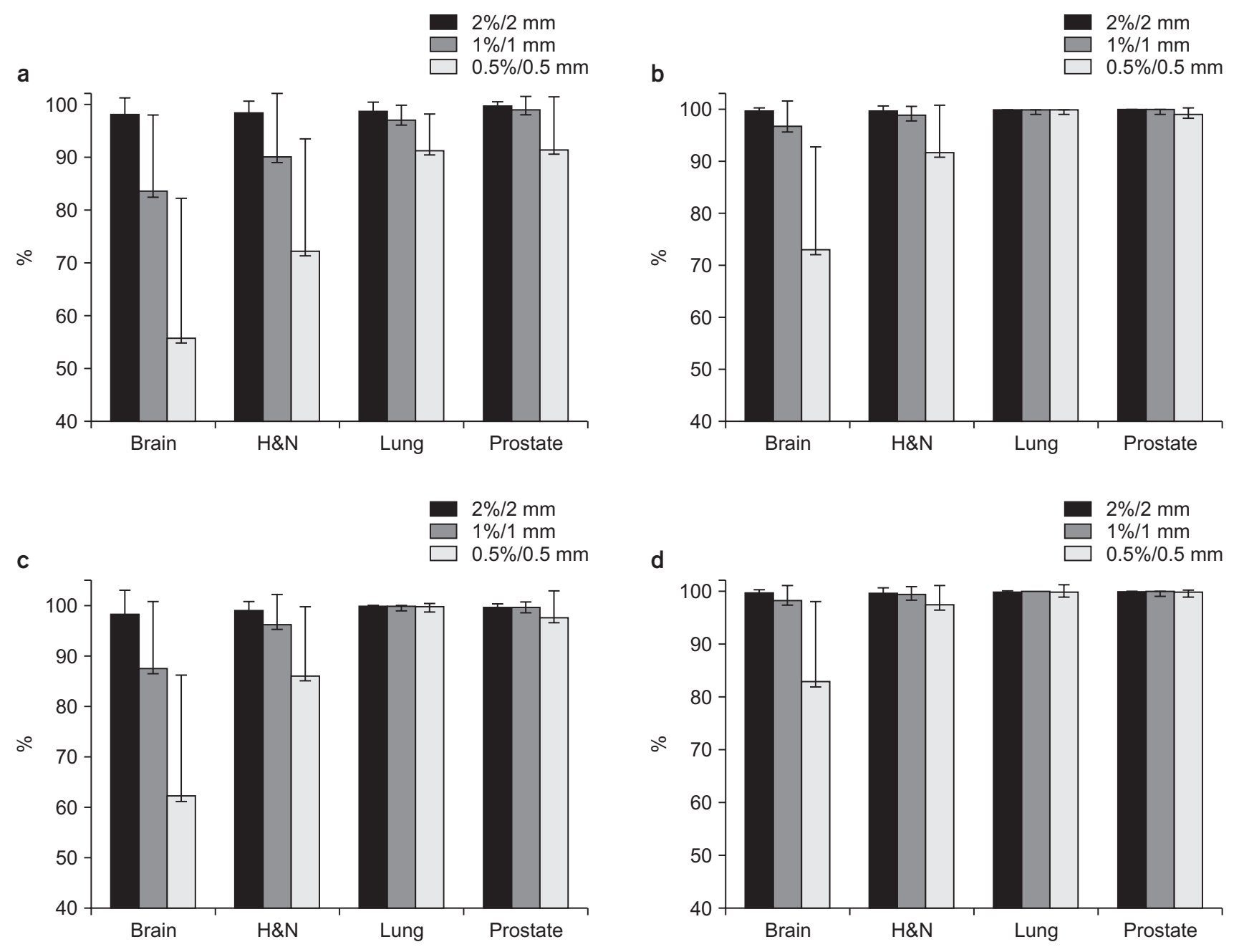

Fig. 4. Local gamma passing rates with various passing criteria calculated from the PTW-VeriSoft ${ }^{\circledR}$ dosimeter measurements are shown for (a) the 80 and $140 \mathrm{kVp}$ plans and (b) the 120 and $140 \mathrm{kVp}$ plans. Global gamma passing rates with various passing criteria calculated from the PTW-VeriSoft ${ }^{\circledR}$ dosimeter measurements are shown for (c) the 80 and $140 \mathrm{kVp}$ plans and (d) the $120 \mathrm{~s}$ and $140 \mathrm{kVp}$ plans. The dosimetric effects for the four treatment sites (brain metastases plans, head and neck cancer plans, lung cancer plans, and prostate cancer plans) were analyzed. The error bars above the histogram indicate the standard deviation.

rates. For the passing criterion of $1 \% / 1 \mathrm{~mm}$, the mean gamma passing rates for the four treatment sites were $87.62 \%$, $96.30 \%, 100 \%$, and $99.62 \%$, respectively $(P<0.001)$. For the passing criterion of $2 \% / 2 \mathrm{~mm}$, the mean gamma passing rates for the treatment sites were $98.43 \%, 98.23 \%, 100 \%$, and $99.85 \%$, respectively ( $P=0.352)$. For 120 and $140 \mathrm{kVp}$ using the passing criterion of $1 \% / 1 \mathrm{~mm}$, the mean gamma passing rates for the four treatment sites were $98.29 \%, 99.37 \%$, $100 \%$, and $100 \%$, respectively $(P=0.025)$. For the passing criterion of $2 \% / 2 \mathrm{~mm}$, the mean gamma passing rates for the four treatment sites were $99.88 \%, 99.79 \%, 100 \%$, and $100 \%$, respectively $(P=0.649)$.

The global gamma passing rates for various passing crite- ria for ED calibrations with different treatment techniques (VMAT and IMAT) were compared, as measured using a dose distribution dosimeter with PTW-VeriSoft ${ }^{\circledR}$ measurements. For the passing criterion of $1 \% / 1 \mathrm{~mm}$, the mean gamma passing rates for the VMAT and IMRT were $97.25 \%$ and $98.70 \%$, respectively $(P=0.15)$. For the passing criterion of $2 \% / 2 \mathrm{~mm}$, the mean gamma passing rates for the VMAT and IMRT were $99.66 \%$ and $99.94 \%$, respectively $(P=0.21)$.

\section{Local gamma passing rates for clinical cases}

Fig. 4 shows the local gamma passing rate for various passing criteria for ED calibrations of 80 and $140 \mathrm{kVp}$ and 

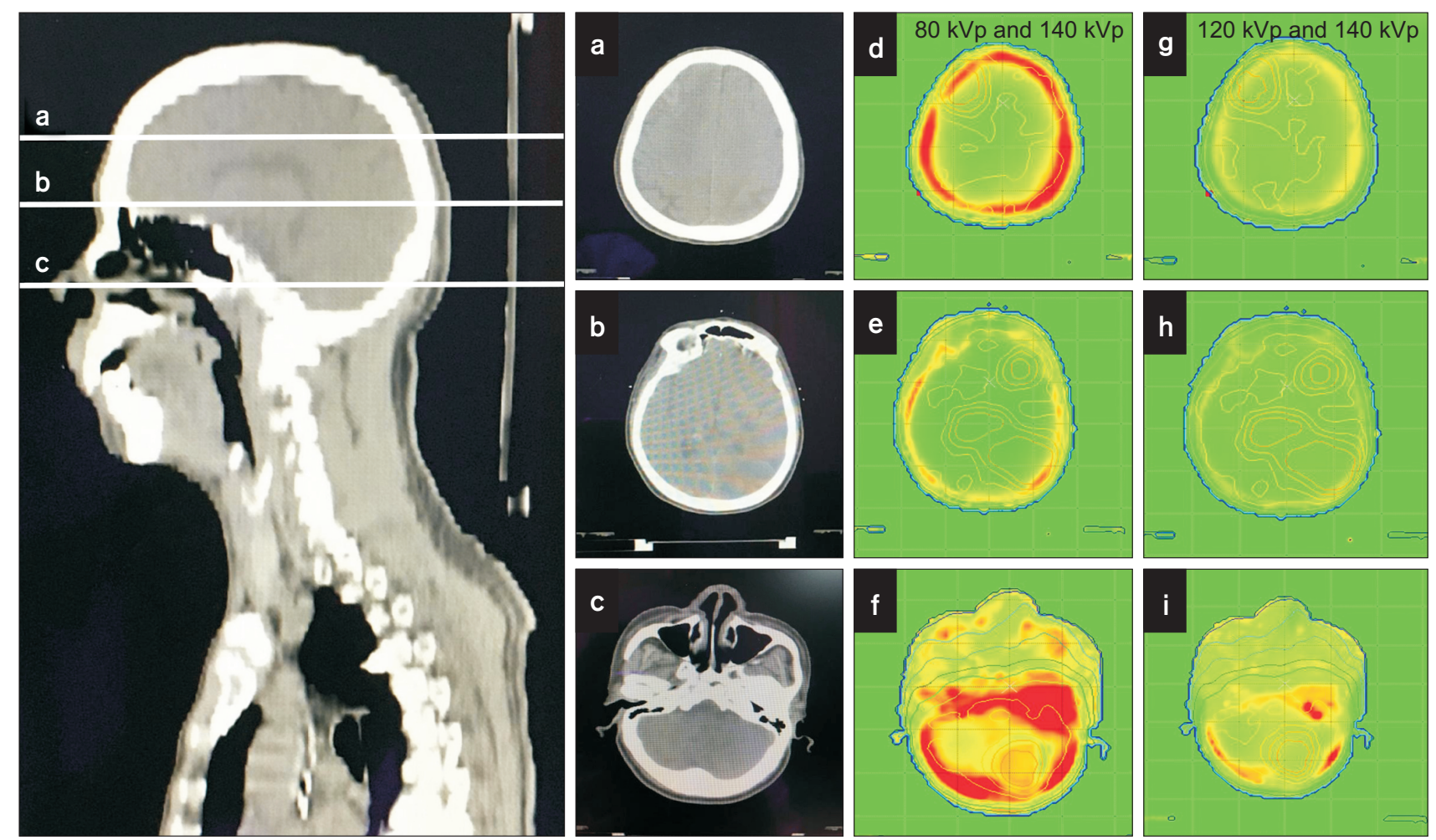

Fig. 5. Sagittal view of a brain metastases treatment plan (a) parallel to the IOM line and across the frontal lobe (the top of the ROI), (b) parallel to the IOM line and across the corpus callosum (the middle of the ROI), and (c) parallel to the IOM line and across the cerebellum (the end of the ROI). The local gamma passing rates for various passing criteria were compared for $1 \% / 1 \mathrm{~mm}$ and show (d-f) ED calibrations of 80 and $140 \mathrm{kVp}$ and (g-i) ED calibrations of 120 and $140 \mathrm{kVp}$. Passing criteria: gamma $\leq 1$. Green 90.0\%-100.0\%, yellow $75.0 \%-90.0 \%$, and red $0.0 \%-75.0 \%$. IOM, inferior orbitomeatal; ROI, circular region of interest; ED, electron density.

120 and $140 \mathrm{kVp}$ for various treatment sites measured using a dose distribution dosimeter with PTW-VeriSoft ${ }^{\circledR}$ measurements.

The ED calibrations for 80 and $140 \mathrm{kVp}$ for brain and H\&N plans were compared to determine the lowest gamma passing rate using a dose distribution dosimeter. For the passing criterion of $1 \% / 1 \mathrm{~mm}$, the mean gamma passing rates for the four treatment sites were $83.53 \%, 90.07 \%, 97.13 \%$, and $99.00 \%$, respectively $(P=0.001)$. For the passing criterion of $2 \% / 2 \mathrm{~mm}$, the mean gamma passing rates for the four treatment sites were $98.11 \%, 98.49 \%, 98.75 \%$, and $99.8 \%$, respectively $(P=0.248)$. Comparison of the results for 120 and 140 $\mathrm{kVp}$ showed that the mean gamma passing rates for the four tumor sites were $96.75 \%, 98.91 \%, 100 \%$, and $100 \%$, respectively, $(P=0.005)$ at the passing criterion of $1 \% / 1 \mathrm{~mm}$. The mean gamma passing rates for the four treatment sites were $99.53 \%, 99.37 \%, 100 \%$, and $100 \%$, respectively, $(P=0.452)$ for the passing criterion of $2 \% / 2 \mathrm{~mm}$.

This study determined that the factors most affecting the
CT number were the tube voltage and treatment site (brain and $H \& N)$. Thus, the tube voltage (80-140 kVp) and treatment site (brain and $H \& N$ ) were used to correct the dose distribution for inhomogeneity.

The sagittal view of the brain metastases treatment plan is parallel to the inferior orbitomeatal (IOM) line and across the frontal lobe (the top of the ROI), across the corpus callosum (the middle of the ROI), and across the cerebellum (the end of the ROI; Fig. 5). The sagittal view of the H\&N tumor treatment plan system is parallel to the IOM line and across the maxillary sinus (the top of the ROI), across the mandible (the middle of the ROI), and across the $\mathrm{C} 7$ spinal disc (the end of the ROI). The gamma index was used to compare the dose distribution (Fig. 6).

The local gamma passing rates for various passing criteria were compared for ED calibrations of $80 \mathrm{kVp}$ and $140 \mathrm{kVp}$ and 120 and $140 \mathrm{kVp}$ for various brain metastases measured using a dose distribution dosimeter. The results are shown in Table 2. The measurement across the frontal lobe 



Fig. 6. Sagittal view of $H \& N$ tumor treatment plan system (a) parallel to the line and across the maxillary sinus (the top of the ROI), (b) parallel to the IOM line and across the mandible (the middle of the ROI), and (c) parallel to the IOM line and across the C7 (the end of the ROI). The local gamma passing rates for various passing criteria were compared for $1 \% / 1 \mathrm{~mm}$ and show (d-f) ED calibrations of 80 and 120 $\mathrm{kVp}$ and (g-i) ED calibrations of 120 and $140 \mathrm{kVp}$. Passing criteria: gamma $\leq 1$. Green $90.0 \%-100.0 \%$, yellow 75.0\%-90.0\%, and red 0.0\%-75.0\%. IOM, inferior orbitomeatal; ROI, circular region of interest; ED, electron density.

Table 2. The local gamma passing rates of the various passing criteria for various brain metastases measured

\begin{tabular}{|c|c|c|c|c|}
\hline \multirow{2}{*}{ Treatment sites } & \multirow{2}{*}{ Passing criterion } & \multicolumn{2}{|c|}{ Local gamma passing rates (\%) } & \multirow{2}{*}{$P$-value } \\
\hline & & 80 and $140 \mathrm{kVp}$ & 120 and $140 \mathrm{kVp}$ & \\
\hline \multicolumn{5}{|l|}{ Brain tumor } \\
\hline \multirow[t]{3}{*}{ Across the frontal lobe } & $2 \% / 2 \mathrm{~mm}$ & $96.67 \pm 4.96$ & $99.53 \pm 0.67$ & 0.009 \\
\hline & $1 \% / 1 \mathrm{~mm}$ & $74.48 \pm 15.39$ & $95.64 \pm 5.95$ & $<0.001$ \\
\hline & $0.5 \% / 0.5 \mathrm{~mm}$ & $42.14 \pm 24.36$ & $64.31 \pm 21.42$ & 0.007 \\
\hline \multirow[t]{3}{*}{ Across the corpus callosum } & $2 \% / 2 \mathrm{~mm}$ & $99.70 \pm 0.46$ & $99.90 \pm 0.16$ & 0.113 \\
\hline & $1 \% / 1 \mathrm{~mm}$ & $96.35 \pm 2.67$ & $98.76 \pm 3.03$ & 0.024 \\
\hline & $0.5 \% / 0.5 \mathrm{~mm}$ & $81.65 \pm 7.42$ & $89.35 \pm 9.15$ & 0.014 \\
\hline \multirow[t]{3}{*}{ Across the cerebellum } & $2 \% / 2 \mathrm{~mm}$ & $98.14 \pm 1.73$ & $99.90 \pm 0.13$ & 0.001 \\
\hline & $1 \% / 1 \mathrm{~mm}$ & $79.32 \pm 6.48$ & $95.50 \pm 3.77$ & $<0.001$ \\
\hline & $0.5 \% / 0.5 \mathrm{~mm}$ & $39.40 \pm 13.51$ & $62.78 \pm 8.15$ & 0.005 \\
\hline
\end{tabular}

Values are presented as mean \pm standard deviation.

dosimeter for the passing criterion of $1 \% / 1 \mathrm{~mm}$ showed mean gamma passing rates of $74.48 \pm 15.39$ and $95.64 \pm 5.95$, respectively $(P<0.001)$. The measurement across the corpus callosum dosimeter for the passing criterion of $1 \% / 1$ mm showed mean gamma passing rates of $96.35 \pm 2.67$ and $98.76 \pm 3.03$, respectively $(P=0.024)$. The measurement across the cerebellum dosimeter for the passing criterion of $1 \% / 1$ mm showed mean gamma passing rates of $79.32 \pm 6.48$ and 
Table 3. The local gamma passing rates of the various passing criteria for various head and neck tumors measured

\begin{tabular}{|c|c|c|c|c|}
\hline \multirow{2}{*}{ Treatment site } & \multirow{2}{*}{ Passing criterion } & \multicolumn{2}{|c|}{ Local gamma passing rates (\%) } & \multirow{2}{*}{$P$-value } \\
\hline & & 80 and $140 \mathrm{kVp}$ & 120 and $140 \mathrm{kVp}$ & \\
\hline \multicolumn{5}{|l|}{ H\&N tumor } \\
\hline \multirow[t]{3}{*}{ Across the maxillary sinus } & $2 \% / 2 \mathrm{~mm}$ & $98.50 \pm 2.21$ & $99.94 \pm 0.78$ & 0.022 \\
\hline & $1 \% / 1 \mathrm{~mm}$ & $88.69 \pm 13.31$ & $98.96 \pm 1.56$ & 0.019 \\
\hline & $0.5 \% / 0.5 \mathrm{~mm}$ & $65.60 \pm 18.28$ & $90.63 \pm 9.35$ & $<0.001$ \\
\hline \multirow[t]{3}{*}{ Across the mandible } & $2 \% / 2 \mathrm{~mm}$ & $97.59 \pm 2.48$ & $99.38 \pm 1.40$ & 0.018 \\
\hline & $1 \% / 1 \mathrm{~mm}$ & $89.15 \pm 9.41$ & $97.94 \pm 2.06$ & 0.001 \\
\hline & $0.5 \% / 0.5 \mathrm{~mm}$ & $69.85 \pm 17.69$ & $89.05 \pm 7.48$ & $<0.001$ \\
\hline \multirow[t]{3}{*}{ Across the C7 spinal segment } & $2 \% / 2 \mathrm{~mm}$ & $99.39 \pm 1.01$ & $100.00 \pm 0.00$ & 0.022 \\
\hline & $1 \% / 1 \mathrm{~mm}$ & $92.21 \pm 13.38$ & $99.85 \pm 0.41$ & 0.030 \\
\hline & $0.5 \% / 0.5 \mathrm{~mm}$ & $80.98 \pm 23.87$ & $95.50 \pm 9.22$ & 0.031 \\
\hline
\end{tabular}

Values are presented as mean \pm standard deviation.

95.50 \pm 3.77 , respectively $(P<0.001)$.

The local gamma passing rates for various passing criteria for ED calibrations of 80 and $140 \mathrm{kVp}$ and 120 and $140 \mathrm{kVp}$ for various $\mathrm{H} \& \mathrm{~N}$ tumors measured using a dose distribution dosimeter are shown in Table 3. The measured value across the maxillary sinus dosimeter for the passing criterion of $1 \% / 1 \mathrm{~mm}$ showed mean gamma passing rates of $88.69 \pm 13.31$ and $98.96 \pm 1.56$, respectively $(P=0.019)$. The measured value across the mandible dosimeter for the passing criterion of $1 \% / 1 \mathrm{~mm}$ showed mean gamma passing rates of $89.15 \pm 9.41$ and $97.94 \pm 2.06$, respectively $(P=0.001)$. The measured value crossing the $\mathrm{C} 7$ spinal segment dosimeter for the passing criterion of $1 \% / 1 \mathrm{~mm}$ showed mean gamma passing rates of $92.21 \pm 13.38$ and $99.85 \pm 0.41$, respectively $(P=0.030)$.

The local gamma passing rates for various passing criteria for ED calibrations with different treatment techniques (VMAT and IMAT) were compared as measured using a dose distribution dosimeter with PTW-VeriSoft ${ }^{\circledR}$ measurements. For the passing criterion of $1 \% / 1 \mathrm{~mm}$, the mean gamma passing rates for the VMAT and IMRT were $96.03 \%$ and $97.24 \%$, respectively $(P=0.40)$. For the passing criterion of $2 \% / 2 \mathrm{~mm}$, the mean gamma passing rates for the VMAT and IMRT were $99.37 \%$ and $99.56 \%$, respectively $(P=0.51)$.

\section{Discussion}

The CT number depends on the CT scanner image protocol conditions. In a previous study, the specifications and standards for a radiotherapy department at a regional hospital in southern Taiwan were assessed [18]. The study found that the smallest deviations in the relationship between the CT number and ED occurred for low ED materials and increased with increasing density. Thomas [19] noted that this deviation was $<1 \%$ for materials with a low ED for different calibration techniques and manufacturers.

The CT number in this study was shown to be stable concerning the different acquisition parameters except for low tube voltage and materials with a higher ED, which resulted in deviations of about $\pm 21.2 \%$.

The results of a similar study assessing the relationship between the CT number and relative ED for various tube voltages reported a maximum difference in the mean dose of $1.1 \%$ [20]. Zurl et al. [11] used different image protocol conditions to assess variation in the $\mathrm{HU}$ of $20 \%$, which resulted in an average dose error of approximately $1.5 \%$. Moreover, Van Dyk et al. [21] and Gershkevitsh et al. [22] showed that a low tube voltage and an increased thickness of the anatomical structure being scanned resulted in a more accurate CT density conversion table that avoided calculation errors $>2 \%$. Kilby et al. [23] showed that radiotherapy at a reasonable dose level was possible for different CT imaging protocol conditions using an average CT density conversion table.

Taken together, these studies support that the factors most affecting the CT number are the tube voltage and treatment site. Given these findings, the gamma index analysis was used to compare the dose distribution in real 
clinical cases. Results showed that the gamma passing rates for ED calibrations of 80,120 , and $140 \mathrm{kVp}$ with the passing criterion of $1 \% / 1 \mathrm{~mm}$ were significant for the four treatment sites. Venselaar et al. [24] showed that the passing criterion of $2 \%$ or $2 \mathrm{~mm}$ in the TPS dose calculations in the central beam was acceptable. This study also noted a maximum difference of only gamma passing rate $<90 \%$ for the passing criterion of 1\%/1 mm for ED calibrations of 80 and 140 $\mathrm{kVp}$ at the treatment site (brain and $\mathrm{H} \& \mathrm{~N}$ ). Thus, the results were clinically insignificant.

$\mathrm{du}$ Plessis et al. [9] reported an average dose error of about $20 \%$ to $70 \%$ in the maxillary sinus region for a head model for different calibration techniques and manufacturers. The anatomical effects of homogeneities must be considered because the brain and $H \& N$ tumor sites are more likely to exhibit an uneven dose distribution. Thus, using the correct CT number-ED conversion table is essential.

Gamma passing rates were used to compare 80,120 , and $140 \mathrm{kVp}$ for brain metastases and H\&N tumors using the passing criteria of $1 \% / 1$ and $2 \% / 2 \mathrm{~mm}$. The results for both regions were statistically significant. The gamma passing rates were $>90 \%$ and $<90 \%$ for the passing criteria of $2 \% / 2$ and 1\%/1 mm, respectively. Zurl et al. [11] used initial calculations for inhomogeneous features of tissue to show that the bone produces more statistically significant results than soft tissue. The skull almost entirely covers the brain tissue for the brain and H\&N tumors at lower tube voltages. Thus, X-ray absorption is important when the X-ray beams traverse inhomogeneous areas. While there are very small differences for the passing criterion of $1 \% / 1 \mathrm{~mm}$ for other treatment sites, the difference is greater for brain and $H \& N$ tumors.

In general, the CT number is most significant for highdensity materials. For CT scanning parameter settings, adjusting the tube voltage has the most significant effect on the brain and H\&N tumor imaging. Yang et al. [25] developed the Swiss Cheese model in 1990 to show that radiological errors can be caused by a series of mistakes. When a medical radiological technologist performs a CT simulation, the operating procedure checklist must be executed to ensure consistency in scanning parameters and to ensure that the correct tube voltage is used to prevent deviation in the TPS dose distribution [25].

\section{Conclusions}

The use of different CT scanning protocols resulted in a variation of up to $21.2 \%$ in the HU values. The local gamma passing rates for various passing criteria for ED calibrations of 80 and $140 \mathrm{kVp}$ were calculated using real patient data, which revealed that brain metastases and $H \& N$ tumors showed a gamma passing rate of $<90 \%$ for the passing criterion of $1 \% / 1 \mathrm{~mm}$. Regarding standardized scanning parameter settings, a specific conversion table needs to be created if the tube voltage in the CT scanner is adjusted. The correct protocol should be used for the corresponding TPS based on the treatment site, especially because this affects the dose distribution for brain metastases and $\mathrm{H} \& \mathrm{~N}$ tumor recognition. Such steps could help reduce systematic errors.

\section{Acknowledgements}

The authors are greatly thankful to Mrs. Lo of Chiu Ho Medical System Co., LTD, Taiwan, for the knowledge on Pinnacle TPS. This study was supported by the Chi Mei Medical Center, Liouying, from January 2020 (grants CLFHR10914).

\section{Conflicts of Interest}

The authors have nothing to disclose.

\section{Availability of Data and Materials}

The data that support the findings of this study are available on request from the corresponding author.

\section{Author Contributions}

Conceptualization: Shu-Chin Yang, Su-Hua Lo, Li-Tsuen Shie, Sung-Wei Lee, and Sheng-Yow Ho. Data curation: ShuChin Yang, Su-Hua Lo, and Li-Tsuen Shie. Formal analysis: Shu-Chin Yang and Su-Hua Lo. Funding acquisition: ShuChin Yang. Investigation: Shu-Chin Yang and Su-Hua Lo. Methodology: Shu-Chin Yang, Su-Hua Lo, Li-Tsuen Shie, Sung-Wei Lee, and Sheng-Yow Ho. Project administration: Shu-Chin Yang and Su-Hua Lo. Resources: Sung-Wei Lee. 
Software: Shu-Chin Yang and Su-Hua Lo. Supervision: ShuChin Yang. Validation: Shu-Chin Yang and Su-Hua Lo. Visualization: Shu-Chin Yang, Su-Hua Lo, Li-Tsuen Shie, SungWei Lee, and Sheng-Yow Ho. Writing-original draft: ShuChin Yang and Su-Hua Lo. Writing-review \& editing: ShuChin Yang, Su-Hua Lo, and Li-Tsuen Shie.

\section{References}

1. Das IJ, Cheng CW, Cao M, Johnstone PA. Computed tomography imaging parameters for inhomogeneity correction in radiation treatment planning. J Med Phys. 2016;41:3-11.

2. Geise RA, McCullough EC. The use of CT scanners in megavoltage photon-beam therapy planning. Radiology. 1977;124:133-141.

3. Goodsitt MM, Christodoulou EG, Larson SC. Accuracies of the synthesized monochromatic CT numbers and effective atomic numbers obtained with a rapid $\mathrm{kVp}$ switching dual energy CT scanner. Med Phys. 2011;38:2222-2232.

4. Lv P, Liu J, Zhang R, Jia Y, Gao J. Combined use of automatic tube voltage selection and current modulation with iterative reconstruction for CT evaluation of small hypervascular hepatocellular carcinomas: effect on lesion conspicuity and image quality. Korean J Radiol. 2015;16:531-540.

5. Guan H, Yin FF, Kim JH. Accuracy of inhomogeneity correction in photon radiotherapy from CT scans with different settings. Phys Med Biol. 2002;47:N223-N231.

6. Davis AT, Palmer AL, Nisbet A. Can CT scan protocols used for radiotherapy treatment planning be adjusted to optimize image quality and patient dose? A systematic review. Br J Radiol. 2017;90:20160406.

7. Cropp RJ, Seslija P, Tso D, Thakur Y. Scanner and kVp dependence of measured CT numbers in the ACR CT phantom. J Appl Clin Med Phys. 2013;14:4417.

8. Skrzyński W, Zielińska-Dabrowska S, Wachowicz M, Slusarczyk-Kacprzyk W, Kukołowicz PF, Bulski W. Computed tomography as a source of electron density information for radiation treatment planning. Strahlenther Onkol. 2010;186:327-333.

9. du Plessis FC, Willemse CA, Lötter MG, Goedhals L. Comparison of the Batho, ETAR and Monte Carlo dose calculation methods in CT based patient models. Med Phys. 2001;28:582-589.
10. Cozzi L, Fogliata A, Buffa F, Bieri S. Dosimetric impact of computed tomography calibration on a commercial treatment planning system for external radiation therapy. Radiother Oncol. 1998;48:335-338.

11. Zurl B, Tiefling R, Winkler P, Kindl P, Kapp KS. Hounsfield units variations: impact on CT-density based conversion tables and their effects on dose distribution. Strahlenther Onkol. 2014;190:88-93.

12. Görlitz E. Dosimetrische und verfahrenstechnische Untersuchungen zur Qualitätssicherung eines Bestrahlungsplanungsprogramms [Thesis]. Hambur: Universität Hamburg; 2006.

13. Low DA, Harms WB, Mutic S, Purdy JA. A technique for the quantitative evaluation of dose distributions. Med Phys. 1998;25:656-661.

14. Cheng A, Harms WB Sr, Gerber RL, Wong JW, Purdy JA. Systematic verification of a three-dimensional electron beam dose calculation algorithm. Med Phys. 1996;23:685693.

15. Shiu AS, Tung S, Hogstrom KR, Wong JW, Gerber RL, Harms WB, et al. Verification data for electron beam dose algorithms. Med Phys. 1992;19:623-636.

16. William BH, Daniel AL, James AP. A quantitative software analysis tool for verifying 3-d dose calculation programs. Int J Radiat Oncol Biol Phys. 1994;30(Suppl 1):187.

17. Ezzell GA, Galvin JM, Low D, Palta JR, Rosen I, Sharpe MB, et al. Guidance document on delivery, treatment planning, and clinical implementation of IMRT: report of the IMRT Subcommittee of the AAPM Radiation Therapy Committee. Med Phys. 2003;30:2089-2115.

18. Fraass B, Doppke K, Hunt M, Kutcher G, Starkschall G, Stern R, et al. American Association of Physicists in Medicine Radiation Therapy Committee Task Group 53: quality assurance for clinical radiotherapy treatment planning. Med Phys. 1998;25:1773-1829.

19. Thomas SJ. Relative electron density calibration of CT scanners for radiotherapy treatment planning. Br J Radiol. 1999;72:781-786.

20. Rhee DJ, Kim S, Jeong DH, Moon YM, Kim JK. Effects of the difference in tube voltage of the CT scanner on dose calculation. J Korean Phys Soc. 2015;67:123-128.

21. Van Dyk J, Barnett RB, Cygler JE, Shragge PC. Commissioning and quality assurance of treatment planning com- 
puters. Int J Radiat Oncol Biol Phys. 1993;26:261-273.

22. Gershkevitsh E, Schmidt R, Velez G, Miller D, Korf E, Yip $\mathrm{F}$, et al. Dosimetric verification of radiotherapy treatment planning systems: results of IAEA pilot study. Radiother Oncol. 2008;89:338-346.

23. Kilby W, Sage J, Rabett V. Tolerance levels for quality assurance of electron density values generated from CT in radiotherapy treatment planning. Phys Med Biol. 2002;47:14851492.
24. Venselaar J, Welleweerd H, Mijnheer B. Tolerances for the accuracy of photon beam dose calculations of treatment planning systems. Radiother Oncol. 2001;60:191-201.

25. Yang SC, Chang YL, Chang LY, Ho SY. The effectiveness of skill learning assessment using direct observation of procedural skills (DOPS) in the training of post-graduate training year and internship students of radiological technologist. C J Radiologic Tech. 2018;42:89-96. 